\title{
Pharmacoinvasive Strategy Versus Lysis and Risk Stratification Strategy in Treatment of Stemi Patients
}

H.M.Kabil, F.M.Swailem, I.S.Alkeshkand and M.M.Othman

Cardiovascular Surgery Dept., Faculty of Medicine, Benha Univ., Benha, Egypt

E-Mail: Mohamed255@gmail.com

\begin{abstract}
Stemi is a clinical syndrome defined by characteristic symptoms of myocardial ischemia in association with persistent electrocardiographic (ECG) ST elevation and subsequent release of biomarkers of myocardial necrosis. Was to detect efficacy and predictors of outcomes of pharmacoinvasive treatment strategy in STEMI patients over reperfusion with thrombolysis only in areas with non-PCI capable centers as in most of hospitals in middle east. A time limited prospective interventional study started in November 2016 till November 2018. Patients divided into two groups, first group 300 patients treated with Lysis full dose TNK followed by risk stratification, the other 300 will go through pharmacoinvasive strategy as stream study by doing routine coronary angiography 3 to 24 hours after successful lysis, if lysis failed in both groups' patient will go directly to rescue PCI. Successful lysis is defined as $>50 \%$ regression of ST segment elevation with pain relief. Lysis associated with more myocardial damage, more Troponin release and less preservation of myocardial contractility function measured by Echo by EF. Pharmacoinvasive strategy significantly reduce risk of reinfarction, need for CABG, PCI, arrhythmia, preservation of EF and at least is as effective as 1ry PCI.
\end{abstract}

Keywords: Pharamcoinvasive , Lysis , MI , STEMI , PCI

\section{Introduction}

STEMI is a clinical syndrome defined by characteristic symptoms of myocardial ischemia in association with persistent electrocardiographic (ECG) ST elevation and subsequent release of biomarkers of myocardial necrosis [1].

STEMI comprises approximately $25 \%$ to $40 \%$ of MI presentations, In-hospital (approximately 5\% to $6 \%$ ) and 1-year (approximately $7 \%$ to 18\%) mortality rates from STEMI also have decreased significantly in association with a substantial increase in the frequency of care that includes GDMT and interventions ("defect-free" care) [1].

In many parts of the world where access to PCIcapable centers 7 days a week and 24 hours a day is available, the preferred approach is for ambulances to bypass a non-PCI capable hospital and directly transport patients to a PPCI facility, Nonetheless, "one size does not fit all" and the preferred reperfusion strategy will depend to a large extent on geographical and logistical constraints such as distance and weather and regional resources [2].

MI is defined pathologically as myocardial cell death due to prolonged ischemia., necrosis progresses from the sub-endocardium to the sub-epicardium over several hours. But biochemical evidence of myocardial cell death due to apoptosis can be detected within 10 minutes of induced myocardial ischemia [3].

Causes of myocardial injury includes could be related to 1ry myocardial ischemia as plaque rupture or thrombus, supply-demand mismatch or multifactorial [3].

Patients can present with prolonged chest pain more than 2 minutes constricting, crushing, compressing or burning radiate to left shoulder, arm, hand, fingers and some times pain is epigastric. Other atypical presentation like syncope, weakness, Dizziness or even silent at all [4].

All Routine blood tests and high sensitive troponin should be carried out [5] .

\section{Management include}

1- Initial diagnosis by ECG within 10 minutes.

2- Routine blood sampling for serum cardiac markers and laboratory.

3- Relief of pain, breathlessness and anxiety.

4- prehospital logistic of care:

$\mathrm{STEMI} \rightarrow$ Time of $\mathrm{PCI} \rightarrow<120 \mathrm{~min} \rightarrow$ 1ry PCI $<$ 90 min reperfusion (wire crossing).

Time of PCI $\rightarrow>120 \mathrm{~min} \rightarrow$ Lysis strategy $<10$ min followed by coronary angiography within $3-24$ hours [6] .

Access route: The preferred Radial than femoral. Rival trial. [7] Preferred stents are DES. EXAMINATION trial. [8] Thrombus aspiration is no more indicated.

Multivessel coronary revascularization could be considered as advised in PRAMI trial and CvLPRIT trial.

Platelet inhibition by DAPT with combination of aspirin and P2Y12 inhibitors, the preferred P2Y12 inhibitors as clopidogrel, ticagrelor, and prasugrel. Enoxaparin is recommended than UFH except with renal impairment. OASIS 6 [9]. Bivalirudin can be used according to HORIZON trial. Fibrinolysis is important reperfusion strategy preserving 30/1000 death and is recommended within 12 hours of symptoms if 1ry PCI can`t be done within $20 \mathrm{~min}$ and prehospital Lysis reduce early mortality by $17 \%$. If Lysis failed it is preferred to shift patient for rescue $\mathrm{PCI}$ even if Lysis is successful (ST segment resolution $>50 \%$ at $60-90$ minutes). A strategy of routine early angiography is recommended if there is no contraindication (STREAM). 
Risk stratification with noninvasive imaging like Echo, MRI, stress MPI, sub-maximum and symptom limited exercise test [10] .

Smoking has strong prothrombotic effect and smoking cessation is potentially the most cost effective of all 2ry prevention measures like Diet, alcohol and weight management keeping BMI $<25$. [11]

Exercise based cardiac rehabilitation should be offered [12].

Antithrombotic therapy with DAPT for a year is recommended according to current - OASIS 7 trial the benefit of mid and long term beta blocker treatment is established [13].

Lipid lowering therapy with high potent statin like atorvastatin $40-80 \mathrm{mg}$ or rosuvastatin $20-40 \mathrm{mg}$ (IMPROVE-IT) trial also proprotein convertase subtilisin/kexin type 9 (PCSK9) improve lipid profile if statin failed (FOURIER) trial. ACE inhibitors are recommended with impaired systolic function.

Complications following ST segment elevation vary from mechanical like left ventricular dysfunction, 2ry Mitral regurgitation, right ventricular involvement, heart failure, free wall ventricular septal \& papillary muscle rupture, pericarditis, pericardial effusion and electrical complications like arrhythmia and conduction dysfunction.

\section{Patients \& methods}

Study was designed as time limited prospective interventional study started in November 2016 till November 2018. Patients divided intyo two groups, first group 300 patients treated with Lysis full dose TNK followed by risk stratification with exercise ECG if their basal ECG is interpretable or stress imaging with nuclear study if not, the other 300 will go through pharmacoinvasive strategy as stream study by doing routine coronary angiography 3 to 24 hours after successful lysis.

\section{Inclusion criteria}

Patients with STEMI eligible for reperfusion with lysis ranging from $18-75$ years old.

\section{Exclusion criteria}

- STEMI patients not eligible for reperfusion with lysis.

- Age Above 75 years old as they should receive half dose Lysis if going for pharmacoinvasive.

- Patients with cardiogenic shock.

- Patient with prior CABG.

\section{Investigations done}

All routine blood tests, Chest Xray, ECG, Echo and Coronary angiography is done through radial and femoral approach with use of 2nd generation of DES.

Follow up of patients over 1, 6 and 12 months.

\section{1ry end point}

Death, MI related death. 2ry end point: cerebrovascular accident.

Study was done to evaluate efficacy of pharmacoinvasive strategy in treatment of STEMI patients.

Statistical analysis of data obtained from database and processed by statistical package for social science version 20 (SPSS 20) program.

Study was carried out in Mubarak AL Kabeer university hospital of Kuwait.

\section{Results}

Statistically significant findings most of patients were male with $\mathrm{p}$ value 0.04 , hypertensive with $\mathrm{p}$ value 0.03 , hyperlipidemic with $\mathrm{p}$ value 0.003 , ischemic heart disease with $\mathrm{p}$ value 0.003 .

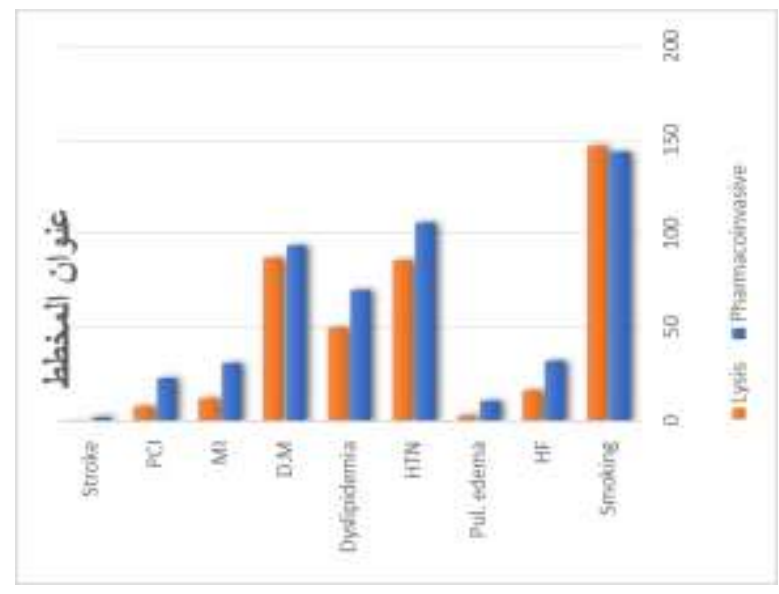

Fig (1) Reperfusion strategy regarding history, presentation \& risk factors.

Troponin elevation was higher in Lysis group than pharmacoinvasive with $\mathrm{p}$ value 0.03 , EF was better in pharmacoinvasive with $p$ value 0.001 , mitral regurgitation was less in pharmacoinvasive with $\mathrm{p}$ value 0.001

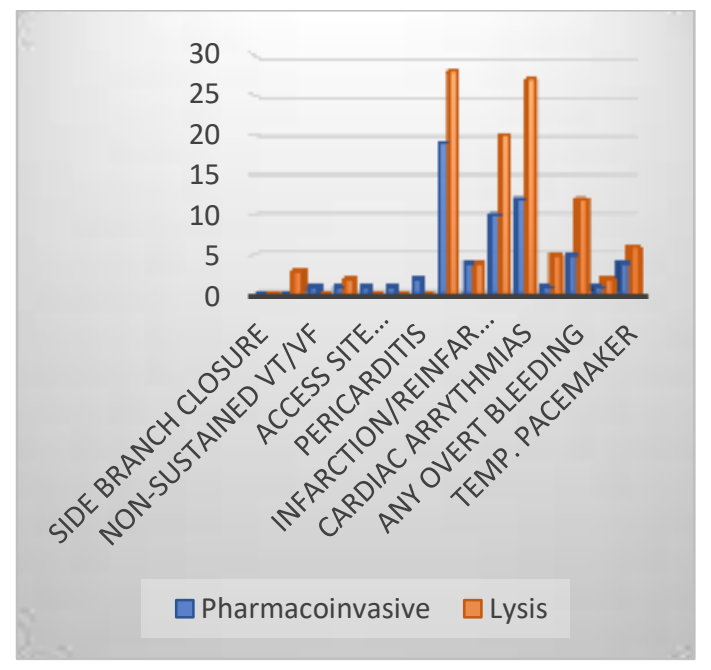

Fig (2) Reperfusion Complication information. 
Reinfarcion was less in pharmacoinvasive with $\mathrm{p}$ value 0.013 , arrhythmias are less in pharmacoinvasive with $\mathrm{p}$ value 0.0001 , stroke was more in lysis with $\mathrm{p}$ value 0.006 , overt bleeding was higher in lysis with $\mathrm{p}$ value 0.0001 .

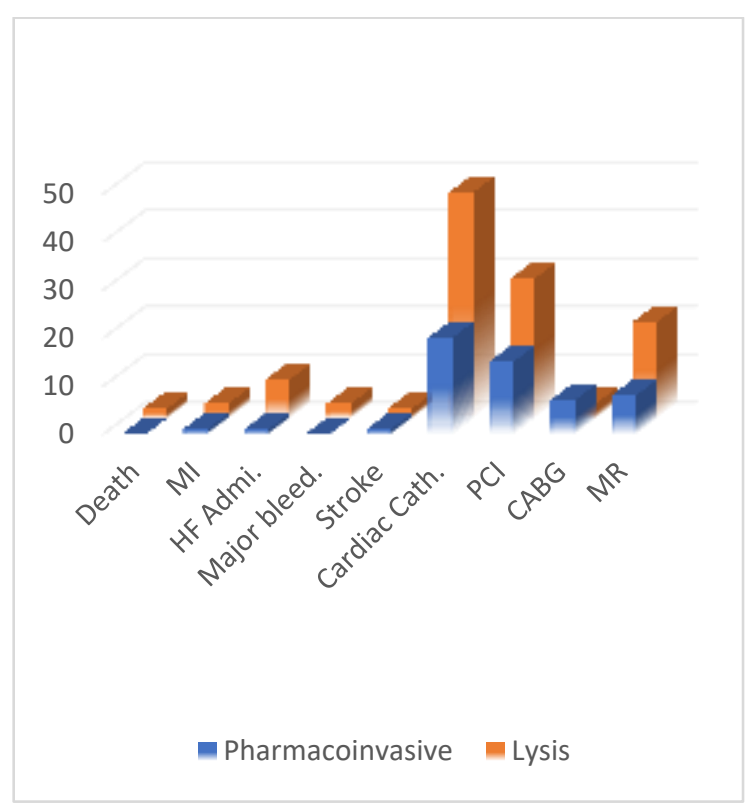

Fig (3) Complication on follow up after 1 month

During follow up HF, major bleeding, stroke, need for coronary angiography, PCI, CABG was significantly higher in Lysis with p values $(0.02,0.07$,
$0.0001,0.0001,0.0001 \& 0.001)$. mitral regurgitation was significantly higher in Lysis with $\mathrm{p}$ value 0.05 and EF was higher in pharmacoinvasive with $\mathrm{p}$ value 0.05 .

\section{Discussion}

Two randomized studies, GRACIA-2 10 and WEST 16, reported comparable efficacy and safety of the pharmacoinvasive strategy versus primary PCI, [6] but these findings could not be considered as being conclusive since the total number of patients randomized in both studies was very low $(n=416)$.

The Strategic Reperfusion Early After Myocardial Infarction (STREAM) study investigated whether prompt thrombolysis at first medical contact, followed by timely angiography or rescue PCI in patients with acute ST-segment elevation myocardial infarction (STEMI) presenting within 3 hours not able to undergo primary PCI within $60 \mathrm{~min}$, is an appropriate and effective reperfusion treatment (pharmacoinvasive strategy) [14].

In our study we found that arrhythmia, bleeding, mitral regurgitation and other complications were significantly higher in Lysis group, but heart failure with presentation was higher in pharmacoinvasive group as patients with heart failure were directed to that strategy but during follow up heart failure was higher in Lysis group, EF was significantly higher in pharmacoinvasive group.

Table(1) Risk stratification in Lysis Group.

\begin{tabular}{lccc}
\hline Variable & Number Of patients & +ve for ischemia & -ve for ischemia \\
\hline Submaximum stress & 200 & 110 & 90 \\
ECG on 5th day & $66 \%$ & $36.66 \%$ & $30 \%$ \\
Stress MPI & 100 & 90 & 10 \\
& $33.3 \%$ & $30 \%$ & $3.3 \%$ \\
Patients had coronary & Total Number & \multicolumn{2}{c}{ Had PCI Done } \\
angiography & 290 & 221 \\
\hline
\end{tabular}

In Lysis group patient risk stratification was carried out and follow up for 12 months significant number of patients had residual ischemia detected as $>5 \%$ defect in stress MPI or +ve Exercise ECG and so went for coronary angiography, PCI \& CABG. Reinfarction was significant higher Lysis group.

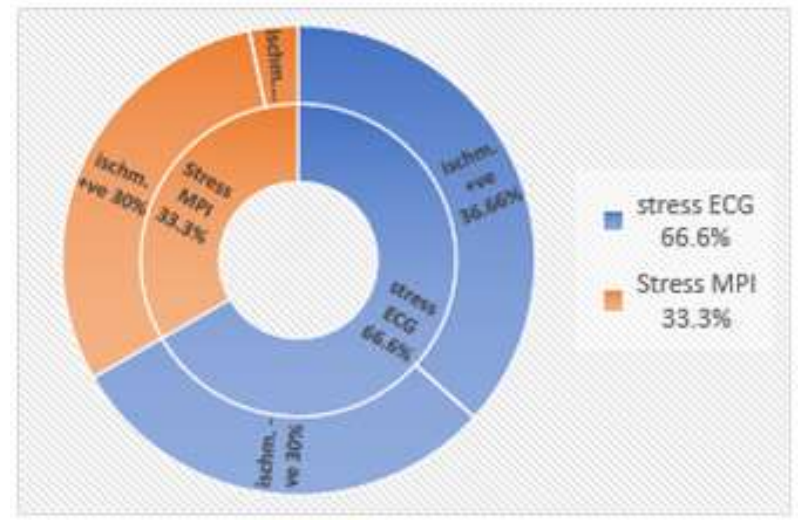

Fig (4) Risk stratification in Lysis Group. 


\section{Conclusion}

The highest incidence regarding age group affected was the age between $45 \& 65$ with increase incidence in the age group below 45 in comparison to before due to bad life style $\&$ increase diabetes incidence.

Lysis is associated with more myocardial damage in comparison to pharmacoinvasive which was proved by more troponin release \& reduced LV EF.

The best access site used was the radial access with least complications \& early mobilization although it was more difficult in cases with complicated \& complex lesions like bifurcational lesions as smaller sheaths are used.

The most common coronary with culprit lesions generally was the LAD, then RCA \& LCX then LM. DES were the most common type of stents used to decrease incidence of instant restenosis \& reinfarction less than BMS used most commonly in the Lysis group as a rescue PCI or CAG \& PCI after risk stratification.

Patients with cardiogenic shock or heart failure should be directed for 1ry PCI or at least pharmacoinvasive strategies. Pharmacoinvasive strategies significantly reduce the risk of reinfarction, need for CAG \& PCI, arrhythmia, preservation of EF $\&$ death than Lysis group, the explanation why the heart failure admissions were in pharmacoinvasive strategy because from the start patients with heart failure or cardiogenic shock were directed for pharmacoinvasive strategy.

Pharmacoinvasive strategy is at least is as effective as 1ry PCI \& all patients presented with STEMI in non PCI capable centers should be transferred for coronary angiography within $3-24$ hours \& even achieved better culprit coronary artery patency.

All patients presented with STEMI \& treated with Lysis in centers far away from PCI capable centers \& can't be shifted within 24 hours should go through risk stratification with submaximal exercise stress ECG on 5th day or fully symptom limited exercise stress ECG after a month or stress MPI or stress ECHO after the 5th day to assess the need for coronary angiography as more than half of patients treated with Lysis had positive tests of ischemia \& had coronary angiography requiring PCI.

PCI capable centers should be available for 24/7 days with well-trained experienced teams for 1ry PCI of all STEMI patients and for shifted patients who had lysis \& keep thrombolytic therapy on the shelf \& in rural areas away from 2ry \& 3ry centers capable of PCI.

\section{Recommendations}

The total number of patients randomized were 600 it was better if more patients can be involved but it was a time limited study.

with low density of PCI-capable hospitals, our study supports the guidelines-recommended option of a pharmacoinvasive approach for early presenters, with administration of thrombolytics followed by expedite interhospital transfer.

Hospital fibrinolysis followed by routine angiography within 6 to 24 hours in stable patients or immediate "rescue PCI "in the remainder is a reasonable alternative to PPCI when delayed by more than 1 hour. Although this study was carefully performed, several issues deserve clarification so that the reader can better put the results in perspective.

our study supports the guidelines-recommended option of a pharmacoinvasive approach for early presenters, with administration of thrombolytics followed by expedite interhospital transfer.

On the contrary, avoidance of thrombolysis and rapid transfer to a PCI facility remains advisable for patients presenting later.

The best access site used was the radial access with least complications \& early mobilization although it was more difficult in cases with complicated \& complex lesions like bifurcational lesions as smaller sheaths are used.

DES were the most common type of stents used incidence of instant restenosis \& reinfarction less than BMS used most commonly in the Lysis group as a rescue PCI or CAG \& PCI after risk stratification.

Patients with cardiogenic shock or heart failure should be directed for 1ry PCI or at least pharmacoinvasive strategies.

Pharmacoinvasive strategy is at least is as effective as 1ry PCI \& all patients presented with STEMI in non-PCI capable centers should be transferred for coronary angiography within $3-24$ hours \& even achieved better culprit coronary artery patency.

All patients presented with STEMI \& treated with Lysis in centers far away from PCI capable centers \& can't be shifted within 24 hours should go through risk stratification with submaximal exercise stress ECG or fully symptom limited exercise stress ECG after a month or stress MPI or stress ECHO after the 5th day to assess the need for coronary angiography as nearly quarter of patients treated with Lysis had positive tests of ischemia \& had coronary angiography requiring PCI.

PCI capable centers should be available for $24 / 7$ days with well trained experienced teams for 1ry PCI of all STEMI patients and for shifted patients who had lysis \& keep thrombolytic therapy on the shelf \& in rural areas away from 2ry \& 3ry centers capable of PCI.

\section{References}

[1] P.T.O'Gara, F.G.Kushner, D.D.Ascheim, ACCF/AHA Guideline for the Management of ST-Elevation Myocardial Infarction: a report of the American College of Cardiology Foundation / American Heart Association Task Force on Practice Guidelines. J Am Coll Cardiol, Vol.61(4), PP. 78-140, 2013.

[2] B.K.Nallamothu,A race for the base: ST-segmentelevation myocardial infarction systems of care in 
low and middle-income countries. Circ Cardiovasc Qual Outcomes, Vol.6,PP.5-6, 2013.

[3] D.S.Ooi, P.A.Isotalo, J.P. Veinot, Correlation of antemortem serum creatine kinase, creatine kinase-MB, troponin $\mathrm{I}$, and troponin $\mathrm{T}$ with cardiac pathology. Clin Chem, PP. 46:338-344, 2000.

[4] B.M.Scirica, M.B.Kadakia, J.A.de Lemos, Association between natriuretic peptides and mortality among patients admitted with myocardial infarction: a report from the ACTION Registry(R)-GWTG. Clin Chem, Vol.59, PP.1205-1214, 2013.

[5] P.Barthel, R.Wensel, A.Bauer, et al. Respiratory rate predicts outcome after acute myocardial infarction: a prospective cohort study. Eur Heart J, Vol.34, PP.1644-1650, 2013.

[6] P.W.Armstrong, A.H.Gershlick, P .Goldstein, R.Wilcox, T.Danays, Y.Lambert, V.Sulimov, F.Rosell Ortiz, M.Ostojic, R.C.Welsh, A.C.Carvalho, J.Nanas, H.R.Arntz, S.Halvorsen, K.Huber, S.Grajek, C.Fresco, E.Bluhmki, A.Regelin, K.Vandenberghe, K.Bogaerts, F.Van de Werf, STREAM Investigative Team. Fibrinolysis or primary PCI in ST-segment elevation myocardial infarction. $\mathrm{N}$ Engl J Med, Vol. 368, PP.1379-87, 2013.

[7] S.S.Jolly, S.Yusuf, J.Cairns, K.Niemela, D.Xavier, P.Widimsky, A.Budaj, M.Niemela, V.Valentin, B.S.Lewis, A.Avezum, P.G.Steg, S.V.Rao, P.Gao, R.Afzal, C.D.Joyner, S.Chrolavicius, S.R.Mehta, RIVAL Trial Group. Radial versus femoral access for coronary angiography and intervention in patients with acute coronary syndromes (RIVAL): a randomised, parallel group, multicentre trial. Lancet, Vol.377(9775), PP.1409-1420, 2011.

[8] M.Sabate , S.Brugaletta, A.Cequier, A.Iniguez, A.Serra, P.Jimenez-Quevedo, V.Mainar, G.Campo, M .Tespili, P.den Heijer, A.Bethencourt, N.Vazquez, G.A.van Es, B.Backx, M.Valgimigli, P.W.Serruys, Clinical outcomes in patients with STsegment elevation myocardial infarction treated with everolimuseluting stents versus bare-metal stents (EXAMINATION): 5-year results of a randomised trial. Lancet.23, Vol.387(10016), PP.357-366, 2016.

[9] S.Yusuf, S.R.Mehta, S.Chrolavicius, R.Afzal, J.Pogue, C.B.Granger, A.Budaj, Peters RJG, Bassand JP, Wallentin L, Joyner C, Fox KAA, OASIS-6 Trial Group. Effects of fondaparinux on mortality and reinfarction in patients with acute ST segment elevation myocardial infarction: the OASIS-6 randomized trial. JAMA, Vol.295(13), PP.1519-1530, 2006.
[10]JR. Timmer, JP .Ottervanger, de Boer MJ, E .Boersma, CL .Grines, CM .Westerhout, RJ.Simes, GrangerCB, ZijlstraF, Primary Coronary Angioplasty vs Thrombolysis-2 Trialists Collaborators G .Primary percutaneous coronary intervention compared with fibrinolysis for myocardial infarction in diabetes mellitus: results from the Primary Coronary Angioplasty vs. Thrombolysis-2 trial. Arch Intern Med; Vol.167(13), PP.1353-1359, 2007.

[11]A.Villella, AP .Maggioni, M .Villella , Prognostic significance of maximal exercise testing after myocardial infarction treated with thrombolytic agents: the GISSI-2 data-base. Gruppo Italiano per lo Studio della Sopravvivenza Nell'Infarto. Lancet; Vol.346 (8974), PP. 523-9, 1995.

[12]S .Chow CK, Jolly, P .Rao-Melacini, KA .Fox, SS .Anand, JP .Yusuf Collet, K .Huber, M .Cohen, U .Zeymer, P .Goldstein, C .Pollack, Jr, J .Silvain, P .Henry, O .Varenne, D .Carrie, P .Coste, M .Angioi, H .Le Breton, G .Cayla, S .Elhadad, E .Teiger, E .Filippi, M .Aout, E .Vicaut, G .Montalescot, ATOLL Investigators. A direct comparison of intravenous enoxaparin with unfractionated heparin in primary percutaneous coronary intervention (from the ATOLL trial). Am J Cardiol; Vol.112(9), PP.1367-1372, PP.2013.

[13]B.Ibanez, C.Macaya, V.Sanchez-Brunete, G.Pizarro, L.Fernandez-Friera, Mateos A, Fernandez-Ortiz A, J.M.Garcia-Ruiz, GarciaAlvarez A, Iniguez A, Jimenez- Borreguero J, Lopez-Romero P, R.Fernandez-Jimenez , J.Goicolea, B.Ruiz-Mateos , T.Bastante, Arias M, Iglesias-Vazquez JA, M.D.Rodriguez, Escalera N, Acebal C, Cabrera JA, J.Valenciano, Perez de Prado A, Fernandez-Campos MJ, I.Casado , J.C.Garcia-Rubira, J.Garcia- Prieto, Sanz-Rosa D, Cuellas C, Hernandez-Antolin R, Albarran A, Fernandez-Vazquez F, de la TorreHernandez JM, Pocock S, Sanz G, Fuster V. Effect of early metoprolol on infarct size in STsegment-elevation myocardial infarction patients undergoing primary percutaneous coronary intervention: the Effect of Metoprolol in Cardioprotection During an Acute Myocardial Infarction (METOCARD-CNIC) trial. Circulation; Vol.128(14), PP.1495-1503, 2013.

[14] T.Y.Wang, B.K.Nallamothu, Krumholz HM, Li S, M.T.Roe, J.G.Jollis, Jacobs AK, D.R. Holmes, E.D.Peterson, H.H.Ting,Association of door-in to door-out time with reperfusion delays and outcomes among patients transferred for primary percutaneous coronary intervention. JAMA, Vol.305, PP.2540-7, 2011. 